\title{
Screening of traditional Chinese medicines with therapeutic potential on chronic obstructive pulmonary disease through inhibiting oxidative stress and inflammatory response
}

\author{
Ming-Xing Zhou' ${ }^{1}$ Xuan Wei ${ }^{2}$, Ai-Ling Li ${ }^{1}$, Ai-Min Wang ${ }^{1}$, Ling-Zi Lu' ${ }^{1}$ Yue Yang ${ }^{1}$, Dong-Mei Ren ${ }^{1}$, Xiao-Ning Wang ${ }^{1}$,
} Xue-Sen Wen ${ }^{1}$, Hong-Xiang Lou ${ }^{1}$ and Tao Shen ${ }^{1 *}$

\begin{abstract}
Background: Chronic obstructive pulmonary disease (COPD) is a major public health problem and gives arise to severe chronic morbidity and mortality in the world. Inflammatory response and oxidative stress play dominant roles in the pathological mechanism of COPD, and have been regarded to be two important targets for the COPD therapy. Traditional Chinese medicines (TCMs) possess satisfying curative effects on COPD under guidance of the TCM theory in China, and merit in-depth investigations as a resource of lead compounds.

Methods: One hundred ninety-six of TCMs were collected, and extracted to establish a TCM extract library, and then further evaluated for their potency on inhibitions of oxidative stress and inflammatory response using $\operatorname{NADP}(H)$ :quinone oxidoreductase (QR) assay and nitric oxide (NO) production assay, respectively.

Results: Our investigation observed that 38 of the tested TCM extracts induced QR activity in hepa 1c1c7 murine hepatoma cells, and 55 of them inhibited NO production in RAW 264.7 murine macrophages at the tested concentrations. Noteworthily, 20 of TCM extracts simultaneously inhibited oxidative stress and inflammatory responses.

Conclusion: The observed bioactive TCMs, particularly these 20 TCMs with dual inhibitory effects, might be useful for the treatment of COPD. More importantly, the results of the present research afford us an opportunity to discover new lead molecules as COPD therapeutic agents from these active TCMs.
\end{abstract}

Keywords: Traditional Chinese medicines, Chronic obstructive pulmonary disease, Oxidative stress, Inflammatory response

\section{Background}

Chronic obstructive pulmonary disease (COPD) is a disease characterized by progressive and not fully reversible airflow limitation, which is associated with abnormal inflammatory response of the lung to noxious particles and gases [1]. Tobacco smoke, indoor and outdoor air

\footnotetext{
* Correspondence: shentao@sdu.edu.cn

'Key Lab of Chemical Biology (MOE), School of Pharmaceutical Sciences,

Shandong University, 44 West Wenhua Road, Jinan 250012, People's Republic of China

Full list of author information is available at the end of the article
}

pollutions, as well as exposure to occupational dust and chemicals are the three dominant risk factors for COPD. It is the fourth leading cause of chronic morbidity and mortality in the United States. On the basis of investigation by the World Bank/World Health Organization, COPD is predicted to rank fifth in 2020 as a worldwide burden of disease. A horrifying fact is that half of global deaths from COPD occur in the Western Pacific Region, with the majority of these existing in China, which might be contributing to high incidence of smoking and severe air pollution in the industrialization advancement [2]. 
Cumulative evidences indicate that inflammatory response, oxidative stress, and protease imbalance play dominant roles in the pathological mechanism of COPD $[3,4]$. Briefly, exogenous irritants and reactive oxygen species (ROS) activate inflammatory cells (e.g. macrophages, neutrophils) and epithelial cells in the respiratory tract that release ROS, inflammatory mediators [e.g. leukotriene B4 (LTB4), interleukin-8 (IL-8), tumor necrosis factor $\alpha$ (TNF $\alpha)$, transforming growth factor- $\beta$ (TGF- $\beta$ )], proteases (e.g. cathepsins, matrix metalloproteinases) $[3,5,6]$. ROS stimulates nuclear factor $k B$ (NF$\mathrm{kB}$ ) and increase the release of inflammatory cytokines, inflammatory mediators promote the production of endogenous ROS, while proteases cause alveolar destruction and mucus secretion. Hence, the synergistic reactions of inflammation, oxidative stress, and protease imbalance amplify pathophysiology of COPD, and inhibitions of these three processes are regarded to be effective strategies for the treatment, as well as drug research and development of COPD [7].

Plenty of traditional Chinese medicines (TCMs) have been used clinically to treat COPD in the form of single or compound prescription under guidance of the TCM theory in China, and demonstrated satisfying curative effects $[8,9]$. Their clinical effectiveness implies that TCM is an important resource of new drugs and/or lead compounds with COPD therapeutic potential. Based on this rationale, we have launched a systemic research on discovering new drugs and lead molecules for COPD treatment from TCM targeting inhibitions of oxidative stress and inflammatory response. We firstly collected and extracted TCM materials to establish a TCM extract library, and then carried out a biological screening of these TCMs using $\mathrm{NADP}(\mathrm{H})$ :quinone oxidoreductase $(\mathrm{QR})$ assay and nitric oxide (NO) production assay to find the TCMs with potential therapeutic effect on COPD.

\section{Methods}

Chemicals

Sulforaphane (SF, purity $>98 \%$ ) was purchased from Sigma-Aldrich (St. Louis, MO, USA). Didox (purity >98 \%) was purchased from MedChem Express (Monmouth Junction, ON, USA). Solvents used for extraction were of analytical grade and obtained from Tianjin Fuyu Chemical Company (Tianjin, China).

\section{Collection and Identification of tested TCMs}

Traditional Chinese medicine (TCM) materials were purchased from the Jinan Jianlian TCM Co. Ltd in Shandong province, Anguo TCM market in Hebei province, and Bozhou TCM market in Anhui Province. These TCMs were identified by Prof. Lan Xiang, School of Pharmaceutical Sciences, Shandong University, through comparing their characteristics in plant morphology and taxonomy with that described in Chinese Pharmacopoeia. Voucher specimens (Voucher ID see Table 1) of TCMs have been deposited at the Laboratory of Pharmacognosy, School of Pharmaceutical Sciences, Shandong University.

\section{Preparations of TCM extractions}

Crushed aerial parts or leaves of plant materials (50 g) were extracted under reflux for $2 \mathrm{~h}$ with $75 \%$ ethanol $(\mathrm{EtOH}, 2 \times 500 \mathrm{~mL})$, and then $\mathrm{EtOH}$ was removed under reduced pressure. The yield of each extract was presented as a percentage of weight of dried plant material, and has been summarized in Table 1 .

\section{Cell cultures}

Hepa 1c1c7 murine hepatoma cells (American Type Culture Collection, ATCC) were maintained in Eagle's minimal essential medium (MEM, Gibco) supplemented with $10 \%$ fetal bovine serum (FBS, Gemini Bio-product). RAW 264.7 murine macrophages (ATCC) were cultured in Dulbecco's Modified Eagle's Medium (DMEM, Gibco) supplemented with $10 \%$ FBS. All cells were incubated at $37{ }^{\circ} \mathrm{C}$ in a humidified incubator containing $5 \% \mathrm{CO}_{2}$.

\section{$\operatorname{NADP}(\mathrm{H})$ : quinone oxidoreductase $(\mathrm{QR})$ assay}

$\operatorname{NADP}(\mathrm{H})$ :quinone oxidoreductase $(\mathrm{QR})$ assay was modified from previously described method [10]. Hepa 1c1c7 cells $\left(1.0 \times 10^{4}\right.$ cells/well $)$ were seeded in 96 -well plates and treated with the indicated doses of tested extracts for $24 \mathrm{~h}$. The medium was decanted, and the cells were incubated with $40 \mu \mathrm{L}$ of lysing solution [0.8\% digitonin and $2 \mathrm{mM}$ EDTA solution ( $\mathrm{pH} 7.8)]$ for $15 \mathrm{~min}$ at $37^{\circ} \mathrm{C}$. Then, $170 \mu \mathrm{L}$ of a complete reaction mixture containing bovine serum albumin, 3-(4,5-dimethylthiazol-2-yl)-2,5diphenyltetrazoliumbromide (MTT), $1.5 \%$ Tween 20, $0.5 \mathrm{M}$ Tris- $\mathrm{HCl}, 7.5 \mathrm{mM}$ flavin adenine dinucleotide (FAD), $150 \mathrm{mM}$ glucose-6-phosphate, 10 units/ $\mu \mathrm{L}$ glucose-6-phosphate dehydrogenase, $50 \mathrm{mM}$ NADP, and $50 \mathrm{mM}$ menadione was added into each well. After incubation for $4 \mathrm{~min}$, a blue color was developed and the reaction was arrested by adding $50 \mu \mathrm{L}$ per well of a $0.3 \mathrm{mM}$ dicoumarol solution ( $\mathrm{pH} 7.4)$. Absorbance was measured at $630 \mathrm{~nm}$ on the Model 680 plate reader (Bio-rad). SF $(2.0 \mu \mathrm{M})$ was adopted as a positive control.

\section{Nitric oxide (NO) production assay}

Inhibition of NO production by LPS-stimulated RAW 264.7 murine macrophages was applied to evaluate antiinflammatory functions of TCM extracts. RAW 264.7 cells $\left(8.0 \times 10^{4}\right.$ cells/well $)$ were seeded in 96-well plates and treated with $1 \mu \mathrm{g} / \mathrm{mL}$ LPS, in the absence or presence of tested TCM extractions for $24 \mathrm{~h}$. Then, $100 \mu \mathrm{L}$ of supernatant was removed to a new 96-well plate and added with $100 \mu \mathrm{L}$ of Griess reagent $(0.1 \%$ 
Table 1 Inhibitions on oxidative stress and inflammation of TCMs evaluated using QR induction and NO production assay

\begin{tabular}{|c|c|c|c|c|c|c|}
\hline No & Plant name & $\begin{array}{l}\text { Part used } \\
\text { in TCM }\end{array}$ & Voucher ID & Yields (\%) & $\begin{array}{l}\text { Induction of QR } \\
\text { activity (MQI) }\end{array}$ & $\begin{array}{l}\text { Inhibition of NO } \\
\text { production (MIR) }\end{array}$ \\
\hline 1 & Acacia catechu (L.f.) Wild. & Branch & 20151128-100-EC & 69.8 & N/D & N/D \\
\hline 2 & Acanthopanax gracilistylus W. W. Smith & Root-bark & 20150802-20-WJP & 9.8 & N/D & $68.0 \%(100)$ \\
\hline 3 & Acanthopanax senticosus (Rupr. et Maxim.) Harms & Rhizome & 20150801-8-CWJ & 7.7 & N/D & $52.0 \%(200)$ \\
\hline 4 & Achyranthes bidentata $\mathrm{BI}$. & Rhizome & 20150801-4-NX & 8.7 & N/D & N/D \\
\hline 5 & Aconitum carmichaeli Debx. & Root & 20151128-58-FZ & 14.7 & N/D & N/D \\
\hline 6 & Acorns tatarinowii Schott & Rhizome & 20151128-30-SCP & 10.5 & N/D & N/D \\
\hline 7 & Adenophora tetraphylla (Thunb.) Fisch. & Root & 20151128-76-SS & 19.1 & N/D & N/D \\
\hline 8 & Agrimonia pilosa Ledeb. & Aerial part & 20151128-31-XHC & 9.8 & N/D & $41.2 \%(200)$ \\
\hline 9 & $\begin{array}{l}\text { Akebia trifoliata (Thunb.) Koidz. subsp. } \\
\text { australis (Diels) T. Shimizu }\end{array}$ & Rattan & 20150802-14-BMT & 11.6 & N/D & N/D \\
\hline 10 & Albizia julibrissin Durazz. & Bark & 20151128-134-HHP & 8.9 & 1.64 fold (200) & N/D \\
\hline 11 & Alisma orientalis (Sam.) Juzep. & Root & 20151128-71-ZX & 13.6 & N/D & $34.2 \%(200)$ \\
\hline 12 & Allium tuberosum Rottl. & Seed & 20150717-4-JCZ & 3.8 & N/D & N/D \\
\hline 13 & Amomum kravanh Pierre ex Gagnep. & Fruit & 20151128-137-DK & 2.1 & N/D & N/D \\
\hline 14 & Amomum villosum Lour. & Fruit & 20150801-9-SR & 8.3 & N/D & N/D \\
\hline 15 & Ampelopsis japonica (Thunb.) Makino & Root & 20151128-127-BL & 10.7 & N/D & N/D \\
\hline 16 & Andrographis paniculata (Burm.f.) Nees & Aerial part & 20151128-83-CXL & 9.9 & 2.04 fold (200) & N/D \\
\hline 17 & Anemarrhena asphodeloides Bge. & Rhizome & $20150802-17-Z M$ & 10.2 & N/D & $41.2 \%(200)$ \\
\hline 18 & Angelica dahurica (Fisch. ex Hoffm.) Benth. et Hook.f. & Root & 20151128-125-BZ & 8.9 & N/D & N/D \\
\hline 19 & Angelica pubescens Maxim. f. biserrata Shan et Yuan & Root & 20150802-16-DH & 25.1 & N/D & N/D \\
\hline 20 & Angelica sinensis (Oliv.) Diels & Root & 20151128-34-DG & 19.4 & 1.41 fold (200) & N/D \\
\hline 21 & Arctium lappa $\mathrm{L}$. & Fruit & 20151128-33-NBZ & 18.4 & N/D & N/D \\
\hline 22 & Areca catechu L. & Peel & 20151128-104-DFP & 2.3 & N/D & N/D \\
\hline 23 & Areca catechu L. & Fruit & 20151128-145-BL & 8.2 & 1.33 fold (25) & N/D \\
\hline 24 & Arisaema erubescens (Wall.) Schott & Tuber & 20151128-23-TNX & 1.2 & 1.36 fold (200) & N/D \\
\hline 25 & Aristolochia debilis Sieb. et Zucc. & Aerial part & 20151128-21-TXT & 3.2 & N/D & N/D \\
\hline 26 & Artemisia argyi Levi. et Vant. & Leaf & 20150716-6-AY & 12.9 & N/D & $86.2 \%(200)$ \\
\hline 27 & Artemisia scoparia Waldst. et Kit. & Aerial part & 20151128-74-YC & 12.8 & 1.48 fold (200) & $38.8 \%(100)$ \\
\hline 28 & $\begin{array}{l}\text { Asarum heterotropoides Fr. Schmldt var. } \\
\text { mandshuricum (Maxim.) Kitag. }\end{array}$ & Root and rhizome & 20151128-72-XX & 11.4 & N/D & N/D \\
\hline 29 & Atractylode lancea (Thunb.) DC. & Rhizome & 20151128-136-CZ & 24.2 & N/D & $52.3 \%(200)$ \\
\hline 30 & Atractylodes macrocephala Koldz. & Rhizome & 20151128-123-BZ & 12.9 & N/D & N/D \\
\hline 31 & Aucklandia lappa Decne. & Root & 20151128-15-MX & 16.3 & 2.31 fold (25) & $94.7 \%(25)$ \\
\hline 32 & Belamcanda chinensis (L.) DC. & Rhizome & 20151128-106-SG & 29.6 & N/D & N/D \\
\hline 33 & Bletilla striata (Thunb.) Reichb.f. & Rhizome & 20150801-12-BJ & 2.1 & N/D & N/D \\
\hline 34 & Bolbostemma paniculatum (Maxim.) Franquet & Tuber & 20151128-5-TBM & 15.1 & N/D & N/D \\
\hline 35 & Callicarpa macrophylla Vahl & Leaf & 20151128-7-DYZZ & 4.3 & N/D & $85.8 \%(200)$ \\
\hline 36 & Cassia angustifolia Vahl. & Leaf & 20150802-25-FXY & 9.4 & 1.54 fold (50) & $79.7 \%(200)$ \\
\hline 37 & Cassia obtusifolia L. & Seed & 20151128-38-JMZ & 7.9 & N/D & N/D \\
\hline 38 & Chaenomeles speciosa (Sweet) Nakai & Fruit & 20151128-24-MG & 30.5 & N/D & N/D \\
\hline 39 & Chrysanthemum morifolium Ramat. & Flower & 20150802-28-JH & 23.3 & N/D & $90.2 \%(200)$ \\
\hline 40 & Cimicifuga heracleifolia Kom. & Rhizome & 20151128-28-SM & 14.1 & 1.95 fold (100) & $86.4 \%(200)$ \\
\hline 41 & Cinnamomum cassia Presl & Branch & 20150717-2-GZ & 5.0 & N/D & N/D \\
\hline 42 & Cirsium japonicum Fisch. ex DC. & Aerial part & 20151128-9-DJ & 6.7 & N/D & N/D \\
\hline
\end{tabular}


Table 1 Inhibitions on oxidative stress and inflammation of TCMs evaluated using QR induction and NO production assay (Continued)

\begin{tabular}{|c|c|c|c|c|c|c|}
\hline 43 & Cirsium setosum (Willd.) M.Bieb. & Aerial part & $20151128-10-X J$ & 12.7 & 1.51 fold (200) & $38.8 \%(200)$ \\
\hline 44 & Cistanchede deserticola Y. C. Ma & Stem & 20150801-7-RCR & 23.6 & N/D & N/D \\
\hline 45 & Citrus aurantium L. & Fruit & 20150801-11-ZQ & 17.0 & N/D & N/D \\
\hline 46 & Citrus limon (L.) Burm. f. & Fruit & 20150716-16-NM & 19.5 & 1.79 fold (200) & N/D \\
\hline 47 & Citrus medica L. var. sarcodac-tylis Swingle & Fruit & 20151128-55-FS & 39.6 & N/D & N/D \\
\hline 48 & Citrus reticulata Blanco. & Pericarp & 20150716-10-СР & 22.0 & N/D & N/D \\
\hline 49 & Clematis armandii Franch. & Rattan & 20150802-12-CMT & 4.5 & N/D & N/D \\
\hline 50 & Codonopsis pilosula(Franch.) Nannf. & Root & 20151128-59-DS & 48.4 & N/D & N/D \\
\hline 51 & Coix lacryma-jobi L. var. ma-yuen (Roman.) Stapf & Seed & 20150716-15-YYR & 6.5 & N/D & N/D \\
\hline 52 & Commelina communis L. & Aerial part & 20151128-88-YZC & 4.4 & N/D & N/D \\
\hline 53 & Coptis chinensis Franch. & Rhizome & 20150802-31-HL & 6.6 & N/D & $57.1 \%(200)$ \\
\hline 54 & Cornus officinalis Sieb. et Zucc. & Fruit & 20150802-27-SZY & 43.6 & N/D & N/D \\
\hline 55 & Crataegus pinnatifida Bge. var. major N. E. Br. & Fruit & 20150716-2-SZ & 34.8 & N/D & N/D \\
\hline 56 & Cremastra appendiculata (D. Don) Makino & Pseudobulb & 20151128-12-SCG & 2.6 & N/D & $61.0 \%(200)$ \\
\hline 57 & Croton tiglium L. & Fruit & 20150802-8-BD & 1.1 & N/D & N/D \\
\hline 58 & Curculigo orchioides Gaertn. & Rhizome & 20151128-121-XM & 4.5 & 1.57 fold (200) & $47.8 \%$ at $(200)$ \\
\hline 59 & Curcuma phaeocaulis Val. & Rhizome & 20150801-20-EZ & 2.6 & N/D & $66.9 \%(25)$ \\
\hline 60 & Curcuma wenyujin Y. H. Chen et C. Ling & Root & $20150802-26-Y J$ & 9.0 & N/D & N/D \\
\hline 61 & Cynanchum atratum Bge. & Root and rhizome & 20151128-40-BW & 23.6 & $N / D$ & N/D \\
\hline 62 & Cynanchum stauntonii (Decne.) Schltr ex Lévl. & Rhizome & 20150801-13-BQ & 10.6. & N/D & N/D \\
\hline 63 & Cynomorium songaricum Rupr. & Stem & 20150716-14-SY & 17.9 & N/D & N/D \\
\hline 64 & Cyperus rotundus L. & Rhizome & 20151128-80-XF & 11.6 & 1.74 fold (200) & N/D \\
\hline 65 & Dendrobium nobile Lindl. & Stem & 20150802-13-MH & 9.0 & N/D & $38.9 \%(200)$ \\
\hline 66 & Dictamnus dasycarpus Turcz. & Velamen & 20150802-4-BXP & 9.0 & N/D & N/D \\
\hline 67 & Dioscorea opposita Thunb. & Rhizome & 20150716-1-SY & 1.7 & N/D & N/D \\
\hline 68 & Dipsacus asperoides C. Y. Cheng et T. M. Ai & Rhizome & 20150716-13-XD & 17.5 & N/D & $38.6 \%(200)$ \\
\hline 69 & Drynaria fortunei (Kunze) J. Sm. & Rhizome & 20151128-78-GSB & 4.8 & N/D & N/D \\
\hline 70 & Eclipta prostrata $\mathrm{L}$. & Aerial part & 20151128-99-MHL & 9.0 & N/D & N/D \\
\hline 71 & Epimedium brevicornum Maxim. & Aerial part & 20150716-9-YYH & 20.5 & N/D & N/D \\
\hline 72 & Equisetum hiemale L. & Aerial part & 20151128-108-MZ & 4.9 & N/D & $41.7 \%(200)$ \\
\hline 73 & Eriocaulon buergerianum Koern. & Flower & 20151128-139-GJC & 7.9 & N/D & N/D \\
\hline 74 & Eucommia ulmoides Oliv. & Root-bark & 20151128-51-DZ & 8.3 & 1.56 fold $(200)$ & $62.5 \%(200)$ \\
\hline 75 & Eugenia caryophyllata Thunb. & Bud & 20151128-1-DX & 27.5 & N/D & N/D \\
\hline 76 & Eupatorium fortunei Turcz. & Aerial part & 20151128-66-PL & 10.1 & N/D & N/D \\
\hline 77 & Euphorbia humifusa Willd. & Whole plant & 20151128-37-DJC & 9.5 & N/D & N/D \\
\hline 78 & Ferula Sinkiangensis K. M. Shen & Resin & 20151128-140-EW & 6.1 & N/D & N/D \\
\hline 79 & Forsythia suspense (Thnub.) Vahl & Fruit & 20151128-53-LQ & 28.3 & N/D & $48.3 \%(200)$ \\
\hline 80 & Fraxinus rhynchophylla Hance & Bark & 20151128-86-QP & 8.0 & N/D & $90.7 \%(200)$ \\
\hline 81 & Fritillaria ussuriensis Maxim. & Bulb & 20151128-118-PBM & 4.1 & N/D & N/D \\
\hline 82 & Ganoderma sinense Zhao, Xu et Zhang & Sporophore & 20150801-2-ZZ & 2.9 & $N / D$ & $N / D$ \\
\hline 83 & Gardenia jasminoides Ellis. & Fruit & 20150717-7-ZZ & 16.1 & N/D & N/D \\
\hline 84 & Gastrodia elata Bl. & Tuber & 20150801-16-TM & 7.5 & N/D & N/D \\
\hline 85 & Glycyrrhiza uralensis Fisch. & Rhizome & 20150716-5-GC & 15.7 & 2.19 fold (100) & $82.9 \%(200)$ \\
\hline 86 & Hippophae rhamnoides L. & Fruit & 20151128-57-S」 & 36.5 & N/D & N/D \\
\hline
\end{tabular}


Table 1 Inhibitions on oxidative stress and inflammation of TCMs evaluated using QR induction and NO production assay (Continued)

\begin{tabular}{|c|c|c|c|c|c|c|}
\hline 87 & Homalomena occulta (Lour.) Schott & Rhizome & 20151128-13-QNJ & 9.3 & N/D & N/D \\
\hline 88 & Hordeum vulgare L. & Fruit & 20151128-135-MY & 11.5 & N/D & N/D \\
\hline 89 & Houttuynia cordata Thunb. & Aerial part & 20150801-18-YXC & 16.7 & N/D & N/D \\
\hline 90 & Illicium difengpi K. I .B. et K. I. M. & Bark & 20151128-132-DFP & 1.9 & 1.52 fold (100) & N/D \\
\hline 91 & Illicium verum Hook. f. & Fruit & 20151128-2-BJHX & 13.3 & N/D & $55.7 \%(200)$ \\
\hline 92 & Inula helenium L. & Root & 20150802-5-TMX & 13.2 & 1.77 fold (12.5) & $100 \%(100)$ \\
\hline 93 & Isatis indigotica Fort. & Root & 20150802-9-BLG & 17.9 & N/D & N/D \\
\hline 94 & Isatis indigotica Fort. & Leaf & 20151128-102-DQY & 13.6 & 1.66 fold $(50)$ & N/D \\
\hline 95 & Kaempferia galanga L. & Rhizome & 20151128-105-SN & 4.7 & N/D & N/D \\
\hline 96 & Kochia scoparia (L.) Schrad. & Fruit & 20150802-23-DFZ & 8.5 & N/D & N/D \\
\hline 97 & Laminaria Japonica Aresch. & Thallus & 20150802-10-KB & 18.5 & N/D & N/D \\
\hline 98 & Lepidium apetalum Willd. & Seed & 20150802-22-TLZ & 2.9 & N/D & N/D \\
\hline 99 & Ligusticum chuanxiong Hort. & Rhizome & 20151128-19-CX & 16.1 & 1.73 fold (200) & $69.0 \%(100)$ \\
\hline 100 & Ligustrum lucidum Ait. & Fruit & 20151128-18-NZZ & 24.2 & N/D & N/D \\
\hline 101 & Lilium lancifolium Thunb. & Leaf & 20151128-32-BH & 4.3 & N/D & N/D \\
\hline 102 & Lindera aggregata (Sims) Kosterm. & Root & 20150801-15-WY & 10.7 & 1.59 fold (200) & N/D \\
\hline 103 & Lithospermum erythrorhizon Sieb. et Zucc. & Root & 20151128-93-ZC & 6.4 & 1.52 fold (50) & $57.1 \%(200)$ \\
\hline 104 & Lobelia chinensi Lour. & Whole plant & 20151128-129-BBL & 23.7 & N/D & N/D \\
\hline 105 & Lonicera hypoglauca Miq. & Flower & 20150801-1-SYH & 31.4 & N/D & N/D \\
\hline 106 & Lonicera japonica Thunb. & Flower & 20150801-3-JYH & 26.7 & N/D & N/D \\
\hline 107 & Lophatherum gracile Brongn. & Stem and leaf & 20151128-91-DZY & 8.6 & N/D & N/D \\
\hline 108 & Lycium barbarum L. & Fruit & 20150801-6-GQ & 11.5 & N/D & N/D \\
\hline 109 & Lycium chinense Mill. & Root-bark & 20150802-21-DGP & 7.5 & N/D & N/D \\
\hline 110 & Lycopodium japonicum Thunb. & Whole plant & 20151128-138-SJC & 22.3 & N/D & $53.0 \%(200)$ \\
\hline 111 & Lycopus lucidusTurcz. var. hirtus Regel & Aerial part & 20151128-70-ZL & 13.5 & N/D & $61.6 \%(200)$ \\
\hline 112 & Lysimachia christinae Hance & Whole plant & 20151128-67-JQC & 11.2 & N/D & N/D \\
\hline 113 & Mahonia bealei (Fort.) Carr. & Stem & 20151128-114-GLM & 6.1 & N/D & N/D \\
\hline 114 & Melia toosendan Sleb. et Zucc. & Fruit & 20151128-107-CLZ & 16.2 & N/D & N/D \\
\hline 115 & Menispermum dauricum DC. & Rhizome & 20151128-120-BDG & 11.0 & N/D & N/D \\
\hline 116 & Mentha haplocalyx Briq. & Aerial part & 20150716-8-BH & 19.1 & N/D & N/D \\
\hline 117 & Mignolia officinalis Rehd. et Wils. & Bark & 20151128-84-HP & 22.9 & N/D & N/D \\
\hline 118 & Misla chinensis Maxim. & Aerial part & 20151128-81-XR & 6.9 & 1.60 fold (100) & N/D \\
\hline 119 & Morinda officinalis How. & Root & 20151128-112-BJT & 28.8 & N/D & N/D \\
\hline 120 & Morus alba L. & Branch & 20151128-142-SZ & 7.4 & 1.37 fold (100) & $61.2 \%(200)$ \\
\hline 121 & Morus alba L. & Fruit & 20151128-143-SS & 29.9 & N/D & N/D \\
\hline 122 & Nardostachys chinensis Batal. & Root and rhizome & 20151128-115-GS & 12.3 & N/D & N/D \\
\hline 123 & Oroxylum inddicum (L.) Vent. & Seed & 20151128-26-MHD & 14.0 & N/D & $85.4 \%(200)$ \\
\hline 124 & Orostachys fimbriatus (Turcz.) Berg. & Aerial part & 20151128-109-WS & 6.3 & $\mathrm{~N} / \mathrm{D}$ & N/D \\
\hline 125 & Paeonia lactiflora Pall. & Rhizome & 20150716-7-BS & 6.4 & N/D & N/D \\
\hline 126 & Panax ginseng C. A. Mey & Rhizome & 20150801-10-SSS & 39.4 & N/D & N/D \\
\hline 127 & Panax quinque folium $\mathrm{L}$. & Root & 20150802-29-XYS & 14.8 & N/D & N/D \\
\hline 128 & Perilla frutescens (L.) Britt. & Leaf & 20150717-6-ZS & 2.8 & 1.73 fold (200) & $57.8 \%(200)$ \\
\hline 129 & Peucedanum praeruptorum Dunn & Root & 20151128-85-QH & 20.6 & N/D & $77.6 \%(200)$ \\
\hline 130 & Phellodendron chinense Schneid. & Tree-bark & 20151128-41-HB & 12.7 & N/D & $\mathrm{N} / \mathrm{D}$ \\
\hline
\end{tabular}


Table 1 Inhibitions on oxidative stress and inflammation of TCMs evaluated using QR induction and NO production assay (Continued)

\begin{tabular}{|c|c|c|c|c|c|c|}
\hline 131 & Phragmites communis Trin. & Root & 20151128-49-LG & 3.8 & $\mathrm{~N} / \mathrm{D}$ & N/D \\
\hline 132 & Physalis alkekengi L. var. franchetii (Mast.) Makino & Calyx & 20150730-2-GJD & 14.2 & 1.79 fold (200) & $91.4 \%(200)$ \\
\hline 133 & Pinellia ternate (Thunb.) Breit. & Tuber & 20151128-130-BX & 0.8 & 1.74 fold (200) & N/D \\
\hline 134 & Piper nigrum L. & Fruit & 20151128-92-HНJ & 5.4 & N/D & N/D \\
\hline 135 & Plantago asiatica $\mathrm{L}$. & Whole plant & 20150716-4-CQC & 13.7 & N/D & N/D \\
\hline 136 & Platycodon grandiflorum (Jacq.) A. DC. & Root & 20151128-87-JG & 33.4 & N/D & N/D \\
\hline 137 & Pogostemon cablin (Blanco) Benth. & Aerial part & 20151128-16-GHX & 5.5 & 1.73 fold (100) & $56.6 \%(200)$ \\
\hline 138 & Polygonatum kingianum Coll. et Hemsl. & Rhizome & 20151128-90-HJ & 6.8 & N/D & N/D \\
\hline 139 & Polygonatum odoratum (Mill.) Druce & Rhizome & 20151128-113-YZ & 21.6 & N/D & N/D \\
\hline 140 & Polygonum aviculare $\mathrm{L}$. & Aerial part & 20151128-89-BX & 10.8 & $\mathrm{~N} / \mathrm{D}$ & N/D \\
\hline 141 & Polygonum cuspidatum Sieb. et Zucc. & Root and rhizome & 20151128-63-HZ & 21.7 & N/D & $60.5 \%(200)$ \\
\hline 142 & Polygonum multiflorum Thunb. & Root & 20151128-54-HSW & 6.8 & N/D & $35.5 \%(200)$ \\
\hline 143 & Polyyala tenuifolia Willd. & Root & 20151128-46-YZ & 34.4 & N/D & N/D \\
\hline 144 & Potentilla chinensis Ser. & Whole plant & 20151128-65-WLC & 8.9 & N/D & N/D \\
\hline 145 & Prunella vulgaris $\mathrm{L}$. & Peel & 20150716-11-XKC & 4.1 & $\mathrm{~N} / \mathrm{D}$ & N/D \\
\hline 146 & Prunus armeniaca L. var. ansu Maxim. & Seed & 20151128-60-KXR & 5.7 & N/D & N/D \\
\hline 147 & Prunus persica (L.) Batsch & Seed & 20151128-61-TR & 3.7 & N/D & N/D \\
\hline 148 & Pseudolarx kaempleri Gord. & Velamen & 20151128-6-TJP & 14.8 & N/D & N/D \\
\hline 149 & Pseudostellaria beterphylla (Miq.) Pax ex Pax et Hoffm. & Root & 20151128-27-TZS & 13.5 & N/D & N/D \\
\hline 150 & Psoralea corylifolia L. & Fruit & 20150801-19-BGZ & 10.2 & N/D & N/D \\
\hline 151 & Pulsatilla chinensis (Bunge) Regel & Root & 20151128-124-BTW & 22.7 & $\mathrm{~N} / \mathrm{D}$ & $\mathrm{N} / \mathrm{D}$ \\
\hline 152 & Punica granatum L. & Peel & 20151128-117-SLP & 31.3 & N/D & N/D \\
\hline 153 & Pyrrosia sheareri (Bak.) Ching & Leaf & 20151128-116-SW & 12.2 & 1.85 fold $(50)$ & N/D \\
\hline 154 & Rabdosia rubescens (Hemsl.) Hara & Aerial part & 20151128-39-DLC & 8.4 & 1.38 fold (100) & $43.2 \%(200)$ \\
\hline 155 & Raphanus sativus L. & Seed & 20150802-30-LFZ & 13.8 & N/D & N/D \\
\hline 156 & Rhaponlicum uniflorum (L.) DC. & Root & 20151128-97-LL & 4.4 & 1.54 fold (200) & N/D \\
\hline 157 & Rheum palmatum L. & Root and rhizome & 20151128-103-DH & 25.6 & N/D & $60.2 \%(200)$ \\
\hline 158 & Rhodiola crenulata (Hook. f. et Thoms.) H. Ohba & Rhizome & 20150801-5-HJT & 13.3 & N/D & $46.3 \%(200)$ \\
\hline 159 & Rosa chinensis Jacq. & Flower & 20151128-110-YJH & 18.9 & N/D & N/D \\
\hline 160 & Rosa laevigata Michx. & Fruit & 20151128-68-JYZ & 25.2 & 1.67 fold $(50)$ & $57 \%(200)$ \\
\hline 161 & Rubia cordifolia L. & Root and rhizome & 20151128-73-QC & 10.0 & N/D & N/D \\
\hline 162 & Salvia miltiorrhiza Bge. & Root and rhizome & 20151128-29-DS & 39.7 & 1.44 fold (100) & $64.5 \%(200)$ \\
\hline 163 & Sanguisorba officinalis L. & Root & 20151128-36-DY & 3.5 & N/D & N/D \\
\hline 164 & Saposhnikovia divaricata (Turcz.) Schischk. & Root & 20150802-19-FF & 15.9 & 1.95 fold (100) & $\mathrm{N} / \mathrm{D}$ \\
\hline 165 & Sareassum pallidum (Turn.) C. Ag. & Frond & $20150802-1-H Z$ & 11.5 & N/D & $\mathrm{N} / \mathrm{D}$ \\
\hline 166 & Sargentodoxa cuneate (Oliv.) Rehd. et Wils. & Rattan & 20151128-8-DXT & 16.9 & N/D & N/D \\
\hline 167 & Scrophularia ningpoensis Hemsl. & Root & 20151128-128-XS & 50.5 & N/D & N/D \\
\hline 168 & Scutellaria baicalensis Georgi. & Rhizome & 20150716-12-HQ & 30.2 & N/D & $87.4 \%(200)$ \\
\hline 169 & Scutellaria barbata D. Don & Whole plant & 20151128-35-BZL & 10.2 & N/D & $59.0 \%(200)$ \\
\hline 170 & Sedum sarmentosum Bunge. & Whole plant & 20151128-64-CPC & 17.5 & N/D & N/D \\
\hline 171 & Selaginella tamariscina (Beauv.) Spring & Whole plant & 20151128-69-JB & 8.9 & N/D & $\mathrm{N} / \mathrm{D}$ \\
\hline 172 & Senecio scandens Buch.-Ham. & Aerial part & 20151128-14-QLG & 11.4 & N/D & $38.1 \%(200)$ \\
\hline 173 & Sesamum indicum L. & Seed & 20150717-3-HZM & 3.3 & N/D & N/D \\
\hline 174 & Siegesbeckia orientalis L. & Aerial part & 20151128-98-XXC & 4.8 & 1.91 fold (200) & $54.9 \%(200)$ \\
\hline
\end{tabular}


Table 1 Inhibitions on oxidative stress and inflammation of TCMs evaluated using QR induction and NO production assay (Continued)

\begin{tabular}{|c|c|c|c|c|c|c|}
\hline 175 & Siphonostegia chinensis Benth. & Whole plant & 20151128-119-BLJM & 9.7 & N/D & $34.6 \%(200)$ \\
\hline 176 & Smilax glabra Roxb. & Rhizome & 20151128-101-TFL & 10.9 & N/D & N/D \\
\hline 177 & Sophora flavescens Ait. & Root & 20151128-62-KS & 21.4 & N/D & N/D \\
\hline 178 & Sophora japonica L. & Flower and bud & 20151128-95-HH & 37.1 & 1.44 fold (200) & N/D \\
\hline 179 & Sparganium stoloniferum Buch.-Ham. & Tuber & 20151128-3-SL & 4.5 & 1.57 fold (200) & N/D \\
\hline 180 & Spatholobus suberectus Dunn & Rattan & 20151128-56-JXT & 5.8 & N/D & N/D \\
\hline 181 & Stemona sessilifolia (Miq.) Miq. & Root & 20150802-24-BB & 30.6 & $\mathrm{~N} / \mathrm{D}$ & N/D \\
\hline 182 & Stephania tetrandra S. Moore & Root & 20151128-43-FJ & 72.9 & $\mathrm{~N} / \mathrm{D}$ & $50.0 \%(200)$ \\
\hline 183 & Sterculia lychnophora Hance & Seed & 20150801-21-PDH & 3.2 & $\mathrm{~N} / \mathrm{D}$ & $30.7 \%(200)$ \\
\hline 184 & Taraxacum mongolicum Hand.-Mazz. & Whole plant & 20150716-3-PGY & 17.2 & N/D & $42.3 \%(200)$ \\
\hline 185 & Taxillus chinensis (DC.) Danser & Aerial part & 20150802-15-SJS & 6.3 & $\mathrm{~N} / \mathrm{D}$ & $N / D$ \\
\hline 186 & Trichosanthes kirilowii Maxim. & Seed & 20131120-1-GL & 20.5 & N/D & $\mathrm{N} / \mathrm{D}$ \\
\hline 187 & Tripterygium wilfordii Hook. f. & Root & 20150802-18-LGT & 8.3 & $\mathrm{~N} / \mathrm{D}$ & N/D \\
\hline 188 & Tussilago farfara $\mathrm{L}$. & Bud & 20150802-6-KDH & 9.9 & 2.38 fold (50) & N/D \\
\hline 189 & Uncaria rhynchophylla (Miq.) Miq. ex Havil. & Stem & 20151128-79-GT & 6.5 & N/D & N/D \\
\hline 190 & Usnea diffracta Vain. & Thallus & 20150802-2-SL & 11.0 & N/D & $44.5 \%(200)$ \\
\hline 191 & Vaccaria segetatis (Neck.) Garcke & Seed & 20151128-20-WBLX & 4.9 & N/D & N/D \\
\hline 192 & Verbena officinalis $\mathrm{L}$. & Aerial part & 20151128-17-MBC & 7.9 & $\mathrm{~N} / \mathrm{D}$ & $\mathrm{N} / \mathrm{D}$ \\
\hline 193 & Viola yedoensis Makino & Whole plant & 20150802-7-ZHDD & 25.6 & $\mathrm{~N} / \mathrm{D}$ & $N / D$ \\
\hline 194 & Xanthium sibiricum Patr. & Fruit & 20151128-47-CEZ & 3.5 & N/D & N/D \\
\hline 195 & Zanthoxylum nitidum (Roxb.) DC. & Root & 20151128-52-LMZ & 6.1 & N/D & $38.2 \%(200)$ \\
\hline 196 & Zanthoxylum schinifoliumSleb. et Zucc. & Peel & 20151128-48-HJ & 21.2 & 1.75 fold (200) & $50.3 \%(200)$ \\
\hline
\end{tabular}

SF $(2.0 \mu \mathrm{M})$ with an approximately 1.7-fold induction was used as a positive control for QR assay; Didox (100 $\mu \mathrm{M})$ with an approximately $60 \%$ inhibition of NO production was adopted as a positive control for NO inhibitory assay; MQI: the maximum folds of QR inducing activity under the tested concentration; MIR: the maximum inhibition rate of NO production under the nontoxic tested concentration; N/D, undetected

naphthylethylenediamine and $1 \%$ sulfanilamide in $5 \%$ $\mathrm{H}_{3} \mathrm{PO}_{4}$ solution) at room temperature for $15 \mathrm{~min}$. Absorbance was measured at $570 \mathrm{~nm}$ on the Model 680 plate reader (Bio-rad). Nitrite concentration was calculated from a $\mathrm{NaNO}_{2}$ standard curve. Didox $(100 \mu \mathrm{M})$ was used as a positive control.

\section{Cell viability assay}

The anti-proliferative effect of TCM extracts on RAW 264.7 cells were simultaneously determined using a 3(4,5-dimthylthiazol-2-yl)-2,5-diphenyltetrazolium bromide (MTT, Sigma) assay. Briefly, $100 \mu \mathrm{L}$ of DMEM media containing $0.4 \%$ MTT were added to each wells, after removing $100 \mu \mathrm{L}$ of supernatant as described in NO production assay. Then, the cells were incubated at $37{ }^{\circ} \mathrm{C}$ for $3 \mathrm{~h}$, and absorbance was measured at $570 \mathrm{~nm}$ on the Model 680 plate reader (Bio-rad).

\section{Statistical analysis}

One way analysis of variance (ANOVA) and post hoc multiple comparison Bonferroni test were applied to compare the significant difference between two groups.
Results are presented as the mean \pm SD. $P<0.05$ was considered to be significant.

\section{Results and Discussion}

To establish a TCM extract library for biological screening, we firstly selected 196 TCMs based on Chinese Pharmacopoeia (Edition 2015) and TCM literatures, and collected TCMs from Jinan local TCM drugstore, as well as the two biggest Chinese TCM markets, Anguo and Bozhou TCM markets. After plant material authentication, TCMs were extracted with $75 \% \mathrm{EtOH}$ to prepare their EtOH extracts, and then the concentrations of $200,100,50,25,12.5,6.25 \mu \mathrm{g} / \mathrm{mL}$ were selected as tested doses for bioassays. Names, origin, extract yields and biological activities of investigated TCMs were summarized in Table 1.

We adopted a bioassay measuring QR activity in hepa 1c1c7 murine hepatoma cells to evaluate the ability of TCM extracts on inhibiting of oxidative stress [10]. Although QR is a phase II detoxification enzyme, it possesses same regulating mechanism with antioxidant enzymes [e.g. glutamate-cysteine ligase, modifier subunit (GCLM) and heme oxygenase-1 (HO-1)], since these 
enzymes are antioxidant response element (ARE)-containing target genes and are mediated by ARE located in their promoter region [11]. Specially, upon the exposure of cells to oxidative stress and/or toxicants, nuclear factor E2-related factor 2 (Nrf2) translocates into the nucleus, binds to the ARE sequence, and activates the transcription of these ARE-target genes [12]. Therefore, QR and antioxidant enzymes (e.g. GCLM and HO-1) possess same responses against endogenous and exogenous insults, which have also been verified by our recent researches $[13,14]$. Considering above mentioned inducing mechanism of QR and antioxidant enzymes, determination of QR activity is a rational and effective method for analyzing the potency of oxidative stress inhibition. In the current study, we normalized the data by setting the untreated control group as 1 , and then the QR inducing activity of tested extracts was represented by the maximum folds of $\mathrm{QR}$ inducing activity (MQI) compared with the untreated control group. SF as a positive control displayed an approximately 1.7 -fold induction at $2.0 \mu \mathrm{M}$. 1.3-fold of $\mathrm{QR}$ inducing activity $(\mathrm{MQI}=1.3)$ under the tested concentrations was adopted as a criterion for bioactive TCM extracts. To be more precise, the level of $\mathrm{QR}$ inducing activity was ranked as the following criteria: strong $(\mathrm{MQI} \geq 1.8)$; moderate $(1.8>\mathrm{MQI} \geq 1.5)$; weak $(1.5>\mathrm{MQI} \geq 1.3)$; undetected $(\mathrm{MQI}<1.3)$.

Ultimately, 38 TCM extracts demonstrated the QR inducing activities with MQI ranging from 1.33- to 2.38- folds under the tested concentrations (Table 1). Of which, eight TCM extracts strongly induced QR activity in hepa $1 \mathrm{c} 1 \mathrm{c} 7$ cells (MQI $\geq 1.8)$, including Andrographis paniculata (aerial part, 16), Aucklandia lappa (root, 31), Cimicifuga heracleifolia (rhizome, 40), Glycyrrhiz uralensis (rhizome, 85), Pyrrosia sheareri (leaf, 153), Saposhnikovia divaricate (root, 164), Siegesbeckia orientalis (aerial part, 174), and Tussilago farfara (bud, 188). Twenty-two extracts are moderate QR inducers (1.8>MQI $\geq 1.5)$, containing Albizia julibrissin (bark, 10), Cassia angustifolia (leaf, 36), Cirsium setosum (aerial part, 43), Citrus limon (fruit, 46), Curculigo orchioides (rhizome, 58), Cyperus rotundus (rhizome, 64), Eucommia ulmoides (root-bark, 74), Illicium difengpi (bark, 90), Inula helenium (root, 92), Isatis indigotica (leaf, 93), Ligusticum chuanxiong (rhizome, 99), Lindera aggregate (root, 102), Lithospermum erythrorhizon (root, 103), Misla chinensis (aerial part, 118), Perilla frutescens (leaf, 128), Physalis alkekengi L. var. franchetii (calyx, 132), Pinellia ternata (tuber, 133), Pogostemon cablin (aerial part, 137), Rhaponlicum uniflorum (root, 156), Rosa laevigata (fruit, 160), Sparganium stoloniferum (tuber, 179), and Zanthoxylum schinifolium (peel, 196). Moreover, eight extracts possessed weak QR inducing effect (1.5 > MQI $\geq 1.3$ ), including Angelica sinensis (root, 20), Areca catechu (fruit, 23), Arisaema erubescens (tuber, 24), Artemisia scoparia (aerial part, 27), Morus alba (branch, 120), Rabdosia rubescens (aerial part, 154), Salvia miltiorrhiza (root and rhizome, 162), and Sophora japonica (flower and bud, 178). QR inducing effects of 38 bioactive TCM extracts in hepa 1c1c7 cells have been detailedly summarized in Additional file 1: Table S1 and Figure S1.

During the chronic inflammation process, excessive NO have been produced and involved in the tissue injury through damages to proteins, lipids, DNA, and the modulation of leukocyte activity [15]. Accordingly, inhibiting NO production is regarded to be an effective strategy for the therapy of inflammation-related diseases. Herein, we detected NO level in LPS-stimulated RAW264.7 macrophages to evaluate anti-inflammatory function of TCM extracts. Cytotoxicities of tested TCM extracts were simultaneously evaluated by a MTT assay to confirm that the decrease of NO production was not attributed to inhibition of cell proliferation. The maximum inhibition rate (MIR) of NO production under the nontoxic tested concentration, which was calculated by comparing the decreased $\mathrm{NO}$ concentration in TCM-treated group with that in LPS-stimulated group, was adopted to evaluate the anti-inflammatory property. Didox with an approximately $60 \%$ inhibition of NO production at $100 \mu \mathrm{M}$ was used as a positive control. The inhibitory potency of TCM extracts on NO production was ranked according to the criteria as follows: strong $(\mathrm{MIR} \geq 80 \%)$; moderate (80 \% > MIR $\geq 50 \%)$; weak (50\% > MIR $\geq 30 \%$ ); undetected (MIR $<30 \%$ ).

Our investigation indicated that 55 TCM extracts inhibited the LPS-induced NO production with MIRs between $30.7 \%$ and $100 \%$ under the tested nontoxic concentrations (Table 1). Thereinto, 11 TCM extracts strongly inhibited NO production in RAW 264.7 cells (MIR $\geq$ $80 \%$ ), including Artemisia argyi (leaf, 26), Aucklandia lappa (root, 31), Callicarpa macrophylla (leaf, 35), Chrysanthemum morifolium (flower, 39), Cimicifuga heracleifolia (rhizome, 40), Fraxinus rhynchophylla (bark, 80), Glycyrrhiza uralensis (rhizome, 85), Inula helenium (root, 92), Oraxylum inddicum (seed, 123), Physalis alkekengi L. var. franchetii (calyx, 132), and Scutellaria baicalensis (rhizome, 168). Moreever, 25 extracts displayed moderate inhibitory effect of NO production $(80 \%>M I R \geq 50 \%)$, and 19 extracts weakly inhibited NO production $(50 \%>$ MIR $\geq 30 \%$ ). Inhibitory effects on NO production of 55 bioactive TCM extracts in RAW 264.7 cells have been detailedly summarized in Additional file 1: Table S1 and Figure S1.

Since oxidative stress and inflammatory response have the synergistic reactions in the pathophysiology of COPD, TCMs having dual inhibitions on the two targets are apt to be the resource for discovering lead molecules $[5,7]$. Our results indicated that the extracts of Artemisia scoparia (aerial part, 27), Aucklandia lappa 
(root, 31), Cassia angustifolia (leaf, 36), Cimicifuga heracleifolia (rhizome, 40), Cirsium setosum (aerial part, 43), Curculigo orchioides (rhizome, 58), Eucommia ulmoides (root-bark, 74), Glycyrrhiza uralensis (rhizome, 85), Inula helenium (root, 92), Ligusticum chuanxiong (rhizome, 99), Lithospermum erythrorhizon (root, 103), Morus alba (branch, 120), Perilla frutescens (leaf, 128), Physalis alkekengi var. franchetii (calyx, 132), Pogostemon cablin (aerial part, 137), Rabdosia rubescens (aerial part, 154), Rosa laevigata (fruit, 160), Salvia miltiorrhiza (root and rhizome, 162), Siegesbeckia orientalis (aerial part, 174), and Zanthoxylum schinifolium (peel, 196) simultaneously inhibited oxidative stress and inflammation (Table 1 and Additional file 1: Table S1). Most of all, both QR inducing effects and NO inhibitory activities of the extracts of Aucklandia lappa (31), Cimicifuga heracleifolia (40), and Glycyrrhiza uralensis (85) are labelled as the level of strong. In addition, the extracts of Inula helenium (92) and Physalis alkekengi L. var. franchetii (132) also demonstrated the potencies that are closed to the strong level.

To our knowledge, this is the first systemic screening of QR inducing extracts from TCMs to discover TCMs with the capacity of inhibiting oxidative stress. Plenty of work on investigation of natural-derived molecules for their regulation on oxidative stress have been carried out, and acquired some active ingredients existed in above evaluated TCMs, such as andrographolide from Andrographis paniculata (16) [16], (Z)-ligustilide from Angelica sinensis (20) [17], dehydroglyasperin C from Glycyrrhiza uralensis (85) [18], isoalantolactone from Inula helenium (92) [19], 2,3'-dihydroxy-4,6'-dimethoxychalcone from Perilla frutescens (128) [20], oridonin from Rabdosia rubescens (154) [21], danshensu and tanshinone I from Salvia miltiorrhiza (162) [22]. These data support our observed QR inducing effects of the active TCMs. More importantly, the majority of QR inducing TCMs tested in present research have still not been phytochemically investigated through targeting oxidative stress inhibition, which affords us an opportunity to discover new lead molecules from them [23].

TCMs have been adopted for the therapy of inflammation-related diseases with a long history in China. Compared with QR inducing assay, NO inhibitory effect assay and other in vitro and in vivo anti-inflammatory models are classical and commonly adopted biological research methods, and accordingly more literatures concerning inflammation of TCMs have been published. Based on our findings, we carried out a systemic search of reported inflammation-related literatures of our observed 55 active TCM extracts, and concluded that: some TCMs have been comprehensively investigated for their antiinflammatory property and resulted in the discovery of diverse types of natural products, covering agrimonolide from
Agrimonia pilosa (8) [24], (-)-nyasol from Anemarrhena asphodeloides (17) [25], alantolactone from Aucklandia lappa (31) [26], berberine from Coptis chinensis (53) [27], forsythiaside from Forsythia suspensa (79) [28], resveratrol from Polygonum cuspidatum (141) [29], etc. Beside these comprehensively investigated molecules, a great deal of constituents have been isolated from these active TCMs, and required further confirmation of their anti-inflammatory function. Meanwhile, a number of TCMs [e.g. Alisma orientalis (11), Equisetum hiemale (72), Cirsium setosum (43)] have not been investigated in the field of inflammation. Significantly, little research on the therapeutic effect of COPD has been performed, and thus these active TCMs are still being researched.

In the present screening assay, we only adopted two typical markers, QR and NO, to evaluate the potential of TCMs as oxidative stress and inflammation inhibitory agents. Based on our preliminary results, active TCM extracts could be subjected to further research in the field of phytochemistry and pharmacology, however, solid evidences on their biological functions are required before a systemic investigation [14]. With regard to the inhibition on oxidative stress, the levels of endogenous glutathione (GSH) and reactive ROS, as well as the protein level of key intracellular redox-balancing protein GCLM, are suggested to be detected to estimate the intracellular redox state and antioxidant capacity when exposed to TCM extracts [30-32]. Concerning the inhibition of inflammation by the active TCMs, the levels of crucial inflammatory mediators in the COPD pathology, including TNF $\alpha$, LTB4, and IL-8, should be determined to confirm their anti-inflammatory potential [33].

Additionally, the pivotal regulators for oxidative stress and inflammation should be sufficiently investigated to verify action of mechanism of the active TCMs. The transcription factor Nrf2 plays a dominant role for regulating oxidative stress. It is ubiquitously expressed in human organs, particularly rich in lung, and counteracts oxidative injury through activating intracellular redox-balancing proteins (e.g. GCLM, GST, HO-1) and up-regulating endogenous antioxidants (e.g. GSH) [11, 34]. NF-kB regulates the expression of proinflammatory genes including cytokines, chemokines, and adhesion molecules, and its inhibition therefore definitely relieves the inflammatory response of COPD [7, 35]. It has also been verified that phosphatidylinositol 3-kinase (PI3K) and mitogen-activated protein kinase (MAPK) are involved in the regulation of inflammatory response [36, 37]. Hence, the further research on active TCM extracts and purified ingredients could focus on their action of mechanism on Nrf2, NF-kB, PI3K, and MAPK signaling pathways, as well as the cross talk between these pathways. 


\section{Conclusion}

Although the present research indicates that some TCMs possessed inhibitory effects on inflammation and oxidative stress, further pharmacological investigations in vitro and in vivo models are warranted. Furthermore, bioassay-guided fractionations and identifications of active ingredients should be launched to help us illustrate the mechanism of these active species, and discover new lead molecules with unknown mechanisms and potent functions on oxidative stress- and inflammationrelated diseases, especially COPD. Accordingly, these results may give new insight in research and development of COPD therapeutic agents.

\section{Additional file}

Additional file 1: Figure S1. $N A D P(H)$ : quinone oxidoreductase $(Q R)$ inducing effects of 38 bioactive TCM extracts in hepa 1c1c7 cells. The QR inducing effect was determined after $24 \mathrm{~h}$ treatment of the hepa $1 \mathrm{clc} 7$ cells in the presence or absence of tested TCMs. The data of the untreated control group was normalized as 1, and then the QR inducing activity of tested extracts was represented by the maximum folds of $Q R$ inducing activity (MQI) compared with the untreated control group. Sulforaphane (SF, $2.0 \mu \mathrm{M}$ ) was used as a positive control. The data are reported the means \pm SD from three independent experiments. Figure S2. Inhibitory effects on NO production of 55 bioactive TCM extracts in RAW 264.7 cells. The NO concentration in the RAW 264.7 cell culture media was determined through the Griess reaction $24 \mathrm{~h}$ after treated in the presence or absence of tested TCMs and lipopolysaccharides (LPS, 1.0 $\mu \mathrm{g} / \mathrm{mL})$. Didox (100 $\mu \mathrm{M})$ was adopted as a positive control. The data are reported the means \pm SD from three independent experiments. The maximum inhibition rates (MIRs) of NO production under the untoxic tested concentration were calculated by comparing the decreased NO concentration in TCM-treated group with that in LPS-stimulated group. Table S1. TCM extracts with QR inducing activity and/or NO inhibitory effect. (DOCX $4312 \mathrm{~kb}$ )

\section{Abbreviations}

ARE: Antioxidant response element; COPD: Chronic obstructive pulmonary disease; GCLM: Glutamate-cysteine ligase, modifier subunit; GSH: Glutathione; GST: Glutathione S transferase; HO-1: Heme oxygenase-1; IL-8: Interleukin-8; LPS: Lipopolysaccharides; LTB4: Leukotriene B4; MAPK: Mitogen-activated protein kinase; MIR: Maximum inhibition rate; MQI: Maximum folds of $Q R$ inducing activity; MTT: 3-(4,5-dimthylthiazol-2-yl)-2,5-diphenyltetrazolium bromide; NF-kB: Nuclear factor kB; Nrf2: Nuclear factor E2-related factor 2; PI3K: Phosphatidylinositol 3-kinase; QR: NADP(H):quinone oxidoreductase; ROS: Reactive oxygen species; SF: Sulforaphane; TCM: Traditional Chinese medicine; TGF- $\beta$ : Transforming growth factor- $\beta$; TNFa: Tumor necrosis factor a

\section{Acknowledgments}

The authors would like to appreciate Profs. Lan Xiang and Hu-Ning Chen, as well as Mr. Yu Zhao in Shandong University for TCM collection and identification.

\section{Funding}

This work was supported by NNSF of China (31470419), NSF of Shandong (ZR2014HM019 and 2015ZRE27209), Science \& Technology Development Plan Project of Shandong (2014GSF118023) and Young Scholars Program of Shandong University (2015WLJH50).

\section{Availability of data and materials}

The datasets during and/or analysed during the current study available from the corresponding author on reasonable request. Moreover, Additional file 1 is available along with the manuscript.

\section{Authors' contributions}

D-MR, $\mathrm{H}-\mathrm{XL}$ and TS conceived and designed the experiments; M-XZ, XW, AMW, L-ZL, YY and X-SW performed the experiments; X-NW and TS analyzed the data; A-LL contributed reagents, materials, and analysis tools; $M-X Z$ and TS wrote the paper. All authors read and approved the final manuscript.

\section{Competing interests}

The authors state no conflict or competing interests are associated with the present study.

\section{Consent for publication}

Not applicable.

\section{Ethics approval and consent to participate}

Not applicable.

\section{Author details}

${ }^{1}$ Key Lab of Chemical Biology (MOE), School of Pharmaceutical Sciences, Shandong University, 44 West Wenhua Road, Jinan 250012, People's Republic of China. ${ }^{2}$ School of Pharmaceutical Sciences, Shandong University of Traditional Chinese Medicine, Jinan, People's Republic of China.

Received: 19 July 2016 Accepted: 10 September 2016

Published online: 13 September 2016

\section{References}

1. Pauwels RA, Buist AS, Calverley PM, Jenkins CR, Hurd S. Global strategy for the diagnosis, management, and prevention of chronic obstructive pulmonary disease. NHLBI/WHO Global Initiative for Chronic Obstructive Lung Disease (GOLD) workshop summary. Am J Respir Crit Care Med. 2001;163:1256-76.

2. Lopez A, Shibuya K, Rao C, Mathers C, Hansell A, Held L, Schmid V, Buist S Chronic obstructive pulmonary disease: current burden and future projections. Eur Respir J. 2006;27:397-412.

3. Barnes PJ, Shapiro S, Pauwels R. Chronic obstructive pulmonary disease: molecular and cellular mechanisms. Eur Respir J. 2003;22:672-88.

4. Barnes PJ, Hansel TT. Prospects for new drugs for chronic obstructive pulmonary disease. Lancet. 2004;364:985-96.

5. Barnes PJ. Mediators of chronic obstructive pulmonary disease. Pharmacol Rev. 2004;56:515-48.

6. Yao H, Rahman I. Current concepts on oxidative/carbonyl stress, inflammation and epigenetics in pathogenesis of chronic obstructive pulmonary disease. Toxicol Appl Pharmacol. 2011;254:72-85.

7. Barnes PJ. Novel approaches and targets for treatment of chronic obstructive pulmonary disease. Am J Respir Crit Care Med. 1999:160:S72-9.

8. Jiang $Y X, W u$ JF, Du J. Survey of study on therapeutic mechanism of chronic obstructive pulmonary disease treated by traditional Chinese medicine. Zhongguo Zhong Xi Yi Jie He Za Zhi. 2005;9:860-4.

9. Gao Z, Li FS, Yang J, Xu D, Yang CH. Investigation on the literature concerning the clinically used traditional Chinese medicines for the treatment of chronic obstructive pulmonary disease in the past ten years. Zhongguo Shi Yan Fang Ji Xue Za Zhi. 2010;16:286-8.

10. Gerhäuser C, You M, Liu J, Moriarty RM, Hawthorne M, Mehta RG, Moon RC, Pezzuto JM. Cancer chemopreventive potential of sulforamate, a novel analogue of sulforaphane that induces phase 2 drug-metabolizing enzymes. Cancer Res. 1997:57:272-8.

11. Lau A, Villeneuve NF, Sun Z, Wong PK, Zhang DD. Dual roles of Nrf2 in cancer. Pharmacol Res. 2008;58:262-70.

12. Bryan HK, Olayanju A, Goldring CE, Park BK. The Nrf2 cell defence pathway: Keap1-dependent and-independent mechanisms of regulation. Biochem Pharmacol. 2013;85:705-17.

13. Shen T, Jiang T, Long M, Chen J, Ren D-M, Wong PK, Chapman E, Zhou B, Zhang DD. A curcumin derivative that inhibits vinyl carbamate-induced lung carcinogenesis via activation of the Nrf2 protective response. Antioxid Redox Signal. 2015;23:651-64.

14. Shen $T$, Chen X-M, Harder B, Long M, Wang X-N, Lou H-X, Wondrak GT, Ren D-M, Zhang DD. Plant extracts of the family Lauraceae: a potential resource for chemopreventive agents that activate the nuclear factor-erythroid 2related factor 2/antioxidant response element pathway. Planta Med. 2014:80:426-34

15. Faro MLL, Fox B, Whatmore JL, Winyard PG, Whiteman M. Hydrogen sulfide and nitric oxide interactions in inflammation. Nitric Oxide. 2014;41:38-47. 
16. Guan S, Tee W, Ng D, Chan T, Peh H, Ho W, Cheng C, Mak J, Wong W. Andrographolide protects against cigarette smoke-induced oxidative lung injury via augmentation of Nrf2 activity. Br J Pharmacol. 2013;168:1707-18.

17. Dietz BM, Liu D, Hagos GK, Yao P, Schinkovitz A, Pro SM, Deng S, Farnsworth NR, Pauli GF, van Breemen RB. Angelica sinensis and its alkylphthalides induce the detoxification enzyme $\mathrm{NAD}(\mathrm{P}) \mathrm{H}$ : quinone oxidoreductase 1 by alkylating Keap1. Chem Res Toxicol. 2008;21:1939-48.

18. Kim HJ, Lim SS, Park IS, Lim JS, Seo JY, Kim JS. Neuroprotective effects of dehydroglyasperin $\mathrm{C}$ through activation of heme oxygenase-1 in mouse hippocampal cells. J Agric Food Chem. 2012;60:5583-9.

19. Seo JY, Park J, Kim HJ, Lee IA, Lim JS, Lim SS, Choi SJ, Park JHY, Kang HJ, Kim JS. Isoalantolactone from Inula helenium caused Nrf2-mediated induction of detoxifying enzymes. J Med Food. 2009;12:1038-45.

20. Izumi Y, Matsumura A, Wakita S, Akagi Kl, Fukuda H, Kume T, Irie K, TakadaTakatori Y, Sugimoto H, Hashimoto T. Isolation, identification, and biological evaluation of Nrf2-ARE activator from the leaves of green perilla (Perilla frutescens var. crispa f. viridis). Free Radic Biol Med. 2012;53:669-79.

21. Du Y, Villaneuva NF, Wang X-J, Sun Z, Chen W, Li J, Lou H, Wong PK, Zhang DD. Oridonin confers protection against arsenic-induced toxicity through activation of the Nrf2-mediated defensive response. Environ Health Persp. 2008;116:1154-61.

22. Tao S, Zheng Y, Lau A, Jaramillo MC, Chau BT, Lantz RC, Wong PK, Wondrak GT, Zhang DD. Tanshinone I activates the Nrf2-dependent antioxidant response and protects against As(III)-induced lung inflammation in vitro and in vivo. Antioxid Redox Signal. 2013;19:1647-61.

23. Kumar H, Kim IS, More SV, Kim BW, Choi DK. Natural product-derived pharmacological modulators of Nrf2/ARE pathway for chronic diseases. Nat Prod Rep. 2014;31:109-39.

24. Chen L, Teng H, Fang T, Xiao J. Agrimonolide from Agrimonia pilosa suppresses inflammatory responses through down-regulation of COX-2/ iNOS and inactivation of NF-kB in lipopolysaccharide-stimulated macrophages. Phytomedicine. 2016;23:846-55.

25. Lee HJ, Li H, Chang HR, Jung H, Lee DY, Ryu JH. (-)-Nyasol, isolated from Anemarrhena asphodeloides suppresses neuroinflammatory response through the inhibition of I-kB alpha degradation in LPS-stimulated BV-2 microglial cells. J Enzyme Inhib Med Chem. 2013;28:954-9.

26. Chun J, Choi RJ, Khan S, Lee DS, Kim YC, Nam YJ, Lee DU, Kim YS, Alantolactone suppresses inducible nitric oxide synthase and cyclooxygenase-2 expression by down-regulating NF-kB, MAPK and AP-1 via the MyD88 signaling pathway in LPS-activated RAW 264.7 cells. Int Immunopharmacol. 2012;14:375-83.

27. Li Z, Geng Y-N, Jiang J-D, Kong W-J. Antioxidant and anti-Inflammatory activities of berberine in the treatment of diabetes mellitus. Evid-Based Compl Alt. 2014;2014:289264.

28. Cheng L, Li F, Ma R, Hu XP. Forsythiaside inhibits cigarette smoke-induced lung inflammation by activation of Nrf2 and inhibition of NF-kappa B. Int Immunopharmacol. 2015;28:494-9.

29. Das S, Das DK. Anti-inflammatory responses of resveratrol. Inflamm Allergy Drug Targets. 2007;6:168-73.

30. Sies H. Glutathione and its role in cellular functions. Free Radic Biol Med. 1999;27:916-21.

31. Lim J, Nakamura BN, Mohar I, Kavanagh TJ, Luderer U. Glutamate cysteine ligase modifier subunit (Gclm) null mice have increased ovarian oxidative stress and accelerated age-telated ovarian failure. Endocrinology. 2015;156: 3329-43.

32. Tong L, Chuang CC, Wu S, Zuo L. Reactive oxygen species in redox cancer therapy. Cancer Lett. 2015;367:18-25.

33. Barnes PJ. Cellular and molecular mechanisms of chronic obstructive pulmonary disease. Clin Chest Med. 2014;35:71-86.

34 Cho HY, Kleeberger SR. Nrf2 protects against airway disorders. Toxicol App Pharmacol. 2010;244:43-56.

35. Lawrence T. The Nuclear Factor NF-kappa B Pathway in Inflammation. Cold Spring Harb Perspect Biol. 2009;1:a001651.

36. Johnson GL, Lapadat R. Mitogen-activated protein kinase pathways mediated by ERK, JNK, and p38 protein kinases. Science. 2002;298:1911-2.

37. Sasaki T, Irie-Sasaki J, Jones RG, Oliveira-dos-Santos AJ, Stanford WL, Bolon B, Wakeham A, Itie A, Bouchard D, Kozieradzki I, Joza N, Mak TW, Ohashi PS, Suzuki A, Penninger JM. Function of PI3Ky in thymocyte development, T cell activation, and neutrophil migration. Science. 2000;287:1040-6.

\section{Submit your next manuscript to BioMed Central and we will help you at every step:}

- We accept pre-submission inquiries

- Our selector tool helps you to find the most relevant journal

- We provide round the clock customer support

- Convenient online submission

- Thorough peer review

- Inclusion in PubMed and all major indexing services

- Maximum visibility for your research

Submit your manuscript at www.biomedcentral.com/submit

) Biomed Central 